\title{
PROCESSAMENTO DE MARCAS PRÉ-ATENTIVO VERSUS ATENTIVO: UM TESTE DAS DIFER ENÇAS NO REGISTRO DOS ESTÍMULOS PELOS INDIVÍDUOS
}

\section{PRE-PROCESSING OF BRANDS VERSUS ATTENTIONAL: A TEST OF THE DIFFERENCES IN THE REGISTRY OF STIMULUS B Y INDIVIDUALS}

\section{Taís Pasquotto Andreoli}

Mestre em Administração pela Faculdade de Economia, Administração e Contabilidade da Universidade de São Paulo - FEAC/USP

E-mail: tais_pa@ hotmail.com (Brasil)

\section{Andres Rodriguez Veloso}

Doutor em Administração pela Faculdade de Economia, Administração e Contabilidade da Universidade de São Paulo - FEAC/USP

Professor da Universidade de São Paulo - USP

E-mail: veloso@usp.br (Brasil)

\section{Leandro Leonardo Batista}

Doutor em Comunicação Social pela University of North Carolina System, UNC SYSTEM, Estados Unidos.

Professor da Escola de Comunicações e Artes da Universidade de São Paulo - ECA/USP

E-mail: leleba@ usp.br (Brasil) 


\section{PROCESSAMENTO DE MARCAS PRÉ-ATENTIVO VERSUS ATENTIVO: UM TESTE DAS DIFERENÇAS NO REGISTRO DOS ESTÍMULOS PELOS INDIVÍDUOS}

\section{RESUMO}

Devido à capacidade limitada dos recursos cognitivos e ao contexto de saturação de estímulos, torna-se provável que muitos dos estímulos disponíveis no ambiente não sejam processados ativamente, de forma consciente, mas sim inconscientemente, ao nível pré-atentivo. Apesar da importância do processamento pré-atentivo, são poucos os estudos que abordam e discutem suas implicações e seu papel na recepção de estímulos. À luz do exposto, o artigo tem como objetivo comparar o processamento pré-atentivo e o atentivo em termos de registro de marcas anunciantes de uma revista, testando, para isso, as memórias implícita e explícita dos indivíduos e sua valoração de marcas. Metodologicamente, procedeu-se, inicialmente, a uma revisão da literatura, focando as seguintes bases conceituais: processo da atenção como constructo complexo, subdividido em pré-atenção e atenção; e o registro das informações à luz do nível de processamento empregado. Tendo por suporte a revisão da literatura, empregou-se o método hipotético-dedutivo, por meio de um experimento 2 (pré-atenção x atenção) x 2 (revista exibida em slide $\mathrm{x}$ impressa). Como contribuição, tem-se a confirmação das hipóteses traçadas: maior incidência de memória explícita observada no processamento atentivo; memória implícita detectada em ambos os processamentos; e efeitos na valoração de marca mais fortes quando houve o processamento pré-atentivo, em detrimento do processamento foi atentivo. Os resultados reforçam a teoria acerca das diferenças entre os registros das informações de acordo com a atenção empregada no processamento, ressaltando, ainda, a superioridade do meio impresso e do processamento pré-atentivo na capacidade de influência dos indivíduos.

Palavras-chave: processamento de marcas; processo da atenção; pré-atenção; registro das informações.

\section{PRE-PROCESSING OF BRANDS VERSUS ATTENTIONAL: A TEST OF THE DIFFERENCES IN THE REGISTRY OF STIMULUS BY INDIVIDUALS}

\section{ABSTRACT}

Due to limited capacity of cognitive resources and to the saturation context of stimuli, it is probable that a lot of available stimuli are not actively processed, in a conscious manner, but unconsciously, at the pre-attentive level. Despite of the importance of pre-attentive processing, there are few studies that address and discuss its implications and function for stimuli reception. This study aims to compare pre-attentive and attentive processing in terms of advertiser brand registry, testing the implicit and explicit memory of individuals in brand valuation. Methodologically, the study proceeded, initially, as a bibliographic survey, focusing on the following conceptual bases: the attention process under a complex perspective, subdivided into pre-attention and attention, and the information registry with regard to the processing level. Supported by the bibliographic survey, the study adopted the hypothetical-deductive method, with the development of a 2 (preattention $\mathrm{x}$ attention) $\mathrm{x} 2$ (magazine shown in slide $\mathrm{x}$ impression) experiment. As a contribution, the study supported the hypotheses: large incidences of explicit memory of advertiser brands were found in attentive processing; implicit memory was found in both methods of processing; and brand valuation effects were higher when the pre-attentive processing occurred, rather than when attentive processing occurred. The results reinforce the theory that registry differences are found according to the attention used for processing and highlighting. Yet, the superiority of the impression of media and pre-attentive processing in the individual influenced capacity.

Key-words: brand processing; process of attention; pre-attention; information registry.

REMark - Revista Brasileira de Marketing, São Paulo, v. 12, n. 4, p. 01-26, out./dez. 2013. 


\section{INTRODUÇÃO}

A maioria das exposições a anúncios de marcas ou produtos ocorre sob condições de mera exposição, ou seja, quando esses estímulos estão disponíveis no ambiente, mas não são necessariamente processados conscientemente pelo individuo (Fang, Singh, \& Ahluwalia, 2007). Isso ocorre porque, em muitas situações, a atenção das pessoas está voltada em outros lugares (objetos-alvo) que não nesses anúncios, o que caracteriza esses estímulos em informações secundárias ou simplesmente informações que não estão sendo buscadas pelos indivíduos (Janis weski, 1993).

Somado a isso, devido à capacidade limitada dos recursos cognitivos, torna-se mais provável que muitos dos estímulos disponíveis no ambiente não sejam processados ativamente, de forma consciente, mas sim pré-conscientemente, ao nível pré-atentivo (Shapiro, Macinnis, \& Heckler, 1994; Rossini \& Galera, 2008). Exemplos desse contexto ocorrem diariamente na vida dos indivíduos: lendo artigos específicos de revistas, mas processando também os anúncios (Shapiro, Macinnis, \& Heckler, 1994); navegando na internet em busca de páginas de interesse, mas processando também os anúncios expostos em suas regiões perimetrais (Yoo, 2008); entre outros.

O processamento pré-atentivo se torna ainda mais usual em ambientes saturados de informações, nos quais muitos estímulos competem por atenção (Janisweski, 1993). Com a revolução e disseminação dos meios de comunicação, que diversificam as fontes de informações bem como ampliam exponencialmente a capacidade de propagação das mesmas (maior rapidez, maior facilidade e menor tempo), são cada vez mais constantes - se não totalitários - os ambientes saturados de informações (Bauman, 2001).

Apesar da importância do processamento pré-atentivo, são poucos - se não raros - os estudos que abordam e discutem as implicações da pré-atenção na recepção de estímulos e seu papel nos efeitos da mera exposição (Milosavljevic; Cerf, 2008). Mais do que isso, nota-se uma equivocada mensuração tanto dos efeitos do processamento atentivo quanto do pré-atentivo, voltada exclusivamente à memória explícita dos indivíduos, em detrimento da memória implícita (Milosavljevic, 2007).

À luz do exposto, o artigo tem como objetivo comparar o processamento pré-atentivo e o atentivo em termos de registro de três marcas anunciantes de uma revista, testando, para isso, as memórias implícita e explícita dos indivíduos e sua valoração de marcas. Metodologicamente, procedeu-se, inicialmente, a uma revisão da literatura, focando as seguintes bases conceituais: processo da atenção como constructo complexo, subdividido em pré-atenção e atenção; e o registro das informações e suas diferenças de acordo com o processamento empregado. Tendo por 
suporte a revisão da literatura, empregou-se o método hipotético-dedutivo, com o desenvolvimento de um experimento com desenho fatorial 2 (processamento pré-atentivo $\mathrm{x}$ atentivo) x 2 (revista exibida em slide ou impressa).

\section{PROCESSO DA ATENÇÃO}

O processamento dos estímulos pode ocorrer, basicamente, em duas etapas diferentes (Tresiman \& Gelade, 1980). A primeira refere-se à pré-atenção ou processamento pré-atentivo, quando uma varredura rápida e geral de todos os estímulos presentes no ambiente é realizada, caracterizando-se por uma extração em paralelo e sem a mobilização de recursos atentivos (Rossini \& Galera, 2008; Rodrigues, Jablonski, \& Assmar, 2008). Em seguida, inicia-se o processo atentivo, que emprega a mobilização serial do foco da atenção a fim de desempenhar uma varredura mais lenta e específica do ambiente, exigindo, para isso, recursos cognitivos superiores (Rossini \& Galera, 2008; Rodrigues, Jablonski, \& Assmar, 2008).

Isso significa dizer que a pré-atenção realiza um trabalho preliminar de analisar todos os estímulos presentes no ambiente, para que, posteriormente, com base nessas informações, a atenção possa dirigir seu foco de análise. Quando o individuo lê uma página de revista, por exemplo, a pré-atenção examina todos os estímulos contidos na página, de forma rápida e geral. De posse dessa informação, a atenção é dirigida ao estímulo de interesse do individuo, geralmente a matéria-principal. As demais áreas, perimetrais, não são atendidas pelo foco da atenção, e continuam a ser processadas de forma pré-atentiva.

\subsection{PRÉ-ATENÇÃo}

O estudo da pré-atenção começou a ser desenvolvido devido à evidência de que informações que estão disponíveis aos indivíduos, mas são ignoradas por eles, podem de fato ser processadas, mesmo que de forma não consciente (Shapiro, Heckler, \& Macinnis, 1997). Ou seja, o fato do individuo decidir não prestar atenção em determinada informação não significa que ela não seja processada por ele, mas sim que ela será processada mesmo sem o conhecimento dele, de forma pré-atentiva. Mais importante, surgem evidências de que o processamento pré-atentivo dos estímulos pode influenciar no julgamento e valoração desses, podendo impactar ainda mais do que o processamento consciente (Shapiro, Heckler, \& Macinnis, 1997; Janisweski, 1993; Yoo, 2008).

Nesse sentido, antes mesmo que a atenção seja ativada, o processamento de estímulos pelos indivíduos já ocorre, de forma pré-atentiva. Assim, previamente à tomada de atenção, o

REMark - Revista Brasileira de Marketing, São Paulo, v. 12, n. 4, p. 01-26, out./dez. 2013. 
processamento pré-atentivo realiza uma varredura geral de todos os estímulos disponíveis, de forma rápida e automática (Janisweski, 1993). Na verdade, a pré-atenção não só antecede a tomada de atenção, como fornece a base de análise para sua direção e seu foco de seleção (Logan, 1992). Ou seja, o processo pré-atentivo é responsável por investigar todos os estímulos disponíveis no ambiente, identificando quais deles são relevantes para futuro detalhamento. Munida desses resultados, a atenção pode ser ativada direcionando e concentrando seus esforços nos estímulos selecionados. Nesse sentido, Wolfe e Horowitz (2003) sugerem que o processo préatentivo deveria, na verdade, ser denominado de processo pré-seletivo da atenção.

De acordo com Janiszewski, (1993), o processo pré-atentivo se caracteriza por um processamento em paralelo, isto é, a pré-atenção realiza uma análise geral e em conjunto de todos os estímulos disponíveis aos órgãos sensoriais. Nessa fase de processamento, não há uma preocupação em distinguir os estímulos relevantes dos irrelevantes, sendo todos processados ao mesmo tempo (Janisweski, 1993). Além disso, o processamento pré-atentivo ocorre de forma automática, não havendo nenhum esforço intencional dos indivíduos nem possibilidade de controle por parte dos mesmos (Wolfe \& Horowitz, 2003). Dessa forma, a pré-atenção trata-se de um processo de codificação mais rápido, que demanda poucos recursos cognitivos.

Uma abordagem bastante recorrente na literatura voltada ao estudo dos processos préatentivos se baseia na teoria da mera exposição, proposta inicialmente por Zajonc, em 1968 (Mandler, Nakamura, \& Van Zandt, 1987; Janis weski, 1993; Lee, 2001; Yoo, 2008; Fang, Singh, \& Ahluwalia, 2007). De forma similar, alguns autores utilizam-se do termo exposição incidental (Shapiro, 1999; Acar, 2007) a fim de estudar os efeitos da mera exposição a um determinado estímulo. De acordo com essa teoria, a mera exposição de um indivíduo a um determinado estímulo, ou seja, a simples disponibilização desse estímulo em um ambiente para que possa ser futuramente percebido ( sem que ele seja atendido pelo foco da atenção), é capaz de causar efeitos, sejam eles implícitos ou explícitos (Zajonc, 1968). Nesse sentido, a teoria da mera exposição afirma que, independente da forma de processamento ocorrida na recepção desses estímulos, ou mesmo se eles chegarão a ser processados de forma ativa pelo cérebro, os estímulos que se encontram disponíveis para acesso impactam e influenciam o indivíduo receptor.

O processo pré-atentivo, ao varrer o ambiente visual como um todo, levantando tantos os estímulos relevantes quando os irrelevantes, grava e armazena um registro de todas as informações analisadas, que pode ser utilizado para referência futura (Janisweski, 1993). Dessa forma, Mandler, Nakamura e Van Zandt (1987) defendem que o processo pré-atentivo interfere em qualquer julgamento posterior ao estímulo, influenciando a maneira como ele é percebido, valorado e interpretado, bem como a forma pela qual seu registro será armazenado.

REMark - Revista Brasileira de Marketing, São Paulo, v. 12, n. 4, p. 01-26, out./dez. 2013. 


\subsection{ATENÇÃO E ATENÇÃO SELETIVA}

De forma geral, Yoo (2008) conceitua a atenção como uma resposta orientada a um determinado estímulo específico. A atenção consiste na tomada de posse pela mente, de forma clara e vívida, de algumas das muitas informações disponíveis no ambiente para futura recepção por parte do indivíduo (James, 1890). Também para De Weerd (2003), a atenção refere-se ao meio pelo qual é processada ativamente e conscientemente uma quantidade limitada de informação frente a muitas presentes no ambiente, somadas àquelas disponíveis através das memórias armazenadas e de outros processos cognitivos.

Dessas definições, infere-se automaticamente o caráter de seletividade da atenção, conceituado como atenção seletiva: focar-se ativa e efetivamente em algumas informações implica afastar-se dos demais estímulos (Sternberg, 2000; De Weerd, 2003). Assim, frente à capacidade limitada dos recursos cognitivos, Sternberg (2000) discute que os fenômenos psicológicos da atenção possibilitam uma melhor utilização desses recursos: ao diminuir a atenção sobre muitos dos estímulos - tanto exteriores (sensações) como interiores (próprios pensamentos e memória) o individuo consegue focar nos estímulos considerados relevantes. Com esse foco dirigido, é maior a probabilidade de resposta rápida e precisa dos estímulos interessantes, aumentando também a probabilidade de que esses registros sejam armazenados e futuramente acionados na memória (De Weerd, 2003).

Recorrente na literatura é a utilização da metáfora do holofote para explicar o modo como a atenção seletiva atua: a luz (atenção) pode ser focada ou dirigida totalmente para uma área de interesse, deixando todo o resto do ambiente na sombra, não focados (Baars, 1998). Trata-se de realçar os estímulos considerados mais interessantes, em detrimento dos demais estímulos disponíveis, que são ignorados ou processados com menor ênfase (Eysenck, 1995). Além disso, a metáfora auxilia na proporção de atenção dirigida, ou seja, quanto mais focado em determinados estímulos, particulares e bem definidos, maior a força da luz (atenção); por outro lado, quanto mais amplo o campo objeto de foco, mais difusa e fraca a iluminação (atenção) a ele dirigida (Eysenck, 1995). Torna-se necessário pontuar que estes estímulos não focados, ainda que não captem o alvo da atenção consciente, podem ser processados de forma pré-atentiva, conforme discutido em tópico anterior.

REMark - Revista Brasileira de Marketing, São Paulo, v. 12, n. 4, p. 01-26, out./dez. 2013. 


\section{REGISTRO DAS INFORMAÇÕES}

Estudos realizados no âmbito da memória sugerem que o registro das informações depende do tipo de tarefa realizada pelo indivíduo, diferenciando o armazenamento de acordo com a complexidade do processamento empregado e da forma de resgate requerido: explícito ou implícito (Yoo, 2008).

A memória explícita se caracteriza por um resgate consciente de alguma lembrança de um momento passado, quando o indivíduo intencionalmente inicia um processo de recuperação de informações previamente armazenadas (Shapiro \& Krishnan, 2001). Sendo assim, as métricas utilizadas para mensurar a memória explícita geralmente empregam métodos diretos, ou seja, são utilizadas medidas que fazem referência direta a um evento passado, exigindo dos indivíduos uma intenção consciente para demonstrar que conseguem se lembrar de determinada lembrança (Yoo, 2005). No contexto da recepção de estímulos, a memória explícita é investigada por meio da capacidade dos indivíduos em evocar o estímulo anteriormente apresentado ou reconhecê-lo frente a estímulos distratores (Milosavljevic, 2007). Por se tratar de um resgate consciente, a memória explícita é atribuída a processamentos mais detalhados, que demandam capacidade cognitiva superior, como é o caso do processamento com foco de atenção dirigido (Yoo, 2008). Dessa forma, espera-se uma influência do tipo de processamento da revista na memória explícita dos indivíduos, mais especificamente, é esperado que os indivíduos que realizarem a leitura da revista de uma forma atentiva respondam por maiores índices de memória explícita do que aqueles que lerem a revista de forma pré-atentiva.

H1: Maiores índices de memória explícita são encontrados quando ocorre o processamento atentivo da revista do que quando o processamento é pré-atentivo.

Por outro lado, a memória implícita diz respeito ao resgate automático de informações previamente armazenadas, não demandando, para is so, a lembrança consciente da informação ou o retorno explícito da experiência (Shapiro, 1999). Dessa forma, o registro de informações na memória implícita é possível em ambos os tipos de processamentos: atentivo e pré-atentivo (Yoo, 2008). A memória implícita é inferida quando há um aprimoramento no desempenho do indivíduo em determinada tarefa subsequente relacionada à prévia exposição e registro do estímulo, ou uma facilitação para que isso ocorra (Yoo, 2005). Outra inferência possível é a maior probabilidade de posterior utilização de informações vistas anteriores, que podem resultar até mesmo em mudanças nas preferências dos indivíduos (Shapiro \& Krishnan, 2001). Como não há um esforço intencional nem consciente de resgate direto da lembrança, nesses casos, são comumente utilizadas métricas

REMark - Revista Brasileira de Marketing, São Paulo, v. 12, n. 4, p. 01-26, out./dez. 2013. 
sem referência direta à prévia exposição, investigando possíveis efeitos de fluência ou priming ou analisando mudanças nas atitudes do individuo perante o estímulo-alvo (Shapiro, Heckler, \& Macinnis, 1997).

Os efeitos de fluência e priming em tarefas subsequentes à prévia exposição se caracterizam pela facilitação do estabelecimento e da ativação do estímulo na memória, incrementando também as chances de futuro processamento ou resgate em uma situação posterior (Monin, 2002). Isso ocorre devido ao registro de uma representação mental dos estímulos previamente analisados, que funciona como uma certa preparação do cérebro para processar estímulos futuros, não só atuando no sentido de facilitar e agilizar os demais processamentos subsequentes, como também aumentando a probabilidade e eficácia desses processamentos analisados (Shapiro, Heckler, \& Macinnis, 1997; Sternberg, 2000).

Isso significa dizer que, por ter sido exposto ao estímulo antes, o cérebro armazenará um registro desse estímulo (representação mental) e, na próxima vez que se deparar com ele, terá maior facilidade em processá-lo. Um exemplo disso utilizado pela literatura é a facilitação encontrada nas tarefas de completar palavras (semelhante ao jogo da forca): os indivíduos acabam utilizando-se de palavras às quais foram expostos anteriormente, mesmo sem ter consciência disso (Shapiro \& Krishnan, 2001; Lee, 2004). Sendo assim, são esperados altos índices de priming em ambos os processamentos (atentivo e pré-atentivo), que, devido à prévia exposição às marcas, facilitará e melhorará o desempenho dos participantes na tarefa de completar palavras.

H2: Com a prévia exposição ocorre o feito priming tanto no processamento atentivo quanto no pré-atentivo.

Complementarmente, Tversky e Kahneman (1974) empregam o termo Heurística da Disponibilidade (availability heuristic) para denotar a facilidade com a qual o estímulo pode ser trazido à mente. Os autores explicam que, quando deparados com tarefas mais trabalhosas, que demandam maior nível de processamento, os indivíduos acabam empregando um número limitado de princípios heurísticos, que decorrem de prévias experiências pessoais (tentativa e erro), a fim de simplificar esses julgamentos. Desse modo, a Heurística da Disponibilidade consiste na simplificação do processamento por meio da utilização de um atalho: confia-se no conhecimento que é facilmente acessível ao invés de procurar e examinar demais alternativas (Tversky \& Kahneman, 1974). Nessa linha de pensamento, Lee e Labroo (2004) afirmam que os indivíduos geralmente baseiam a valoração de um estímulo não apenas nas informações que estão disponíveis

REMark - Revista Brasileira de Marketing, São Paulo, v. 12, n. 4, p. 01-26, out./dez. 2013. 
sobre o mesmo, mas também - e principalmente - na acessibilidade desse estímulo, entendida como a facilidade com que associações ou interpretações surgem à mente.

Como resultado dos efeitos de fluência ou facilidade no processamento do estímulo, o estímulo passa a ser valorado como mais positivo, mesmo que isso ocorra sem a intenção ou consciência por parte do indivíduo (Zajonc, 1968). Na verdade, é esperado que o estímulo seja valorado mais positivamente quando não há consciência dos indivíduos acerca da prévia exposição (processamento pré-atentivo) do que quando eles têm conhecimento dela (processamento atentivo) (Bornstein \& D'Agostinho, 1994). Isso acontece porque, no segundo caso, os indivíduos, tendo consciência do estímulo e de sua prévia exposição, engajam em um processo de correção, interpretando e revisando a fluência/facilidade no processamento do estímulo. Com isso, a valoração positiva atribuída ao estímulo é corrigida, fato que não ocorre na pré-atenção, quando os indivíduos não têm conhecimento dos estímulos processados (Shapiro, Heckler, \& Macinnis, 1997). Dessa forma, espera-se que haja maiores níveis de positividade atribuídos às marcas anunciantes quando o processamento for pré-atentivo, em detrimento do atentivo.

H3: Maiores índices de valoração de marca em termos de positividade são encontrados quando ocorre o processamento pré-atentivo da revista do que quando o processamento é atentivo.

Por ser valorado mais positivo, o estímulo passa a ser interpretado co mo mais atrativo ou agradável, condição pré-requisitada para uma futura preferência (Janisweski, 1993). Além disso, o individuo desenvolve sentimentos de intimidade e proximidade com o estímulo, devido ao senso de familiaridade atribuído ao mesmo (Janisweski, 1993). Esse senso de familiaridade, somado à preferência pelo estímulo, facilita a incorporação do estímulo no conjunto de consideração do indivíduo, aumentando as chances desse ser acatado em um momento futuro de tomada de decisão, principalmente se esse processo for inconsciente (ou seja, sem que haja possibilidade de correção dessa valoração) (Shapiro, Heckler, \& Macinnis, 1997; Holden \& Vanhuele, 1999).

Assim, os autores propõem que, ao ativar o estímulo na memória e facilitar seu acesso, aumenta-se a probabilidade de que esse estímulo seja incluído no conjunto de consideração do indivíduo. Isso decorre da tentativa de minimizar os esforços envolvidos na tomada de decisão: ao escolher um estímulo que parece mais positivo, contribui-se tanto para agilizar o processo decisório (diminuindo tempo gasto) quanto para torná-lo mais eficaz (menor uso de recursos cognitivos) (McDonald \& Sharp, 2003).

O conjunto de consideração do individuo consiste no conjunto de estímulos que serão considerados em futura tomada de decisão, geralmente representando apenas uma fração da soma 
de todos os estímulos disponíveis (Hauser \& Wenerfelt, 1990). Nesse sentido, os autores defendem que esse conjunto difere-se também do conjunto de consciência do indivíduo, já que considera apenas uma parte de todos os estímulos dos quais o individuo tem conhecimento. Reilly e Parkinson (1985) postulam que essa inclusão do estímulo no conjunto de consideração do individuo se faz condição necessária para a futura seleção do estímulo e concretização da decisão. Com base nessa discussão, espera-se também aqui uma preponderância do processamento préatentivo sobre o atentivo, com maiores índices de intenção de compra das marcas atribuídos ao primeiro caso em detrimento do segundo.

H4: Maiores índices de valoração de marca em termos de intenção de compra da marca são encontrados quando ocorre o processamento pré-atentivo da revista do que quando o processamento é atentivo.

Campbell e Keller (2003) argumentam, ainda, que quando expostos a estímulos familiares, os consumidores têm maior propensão em atualizar o prévio conhecimento armazenado no cérebro. Além disso, segundo os autores, nesses casos o risco percebido pelos consumidores é menor, tornando maior a probabilidade de um processamento mais favorável do estímulo, ou seja, esses estímulos familiares são processados com menor disposição de resistência ou contraargumentação por parte dos indivíduos receptores. Nesse sentido, Bornstein e D’Agostinho (1994) defendem que, de forma geral, o processamento dos estímulos de forma pré-atentiva consegue impactar mais os indivíduos receptores do que quando o processamento ocorre de modo atentivo. Com isso, espera-se que, de uma forma geral, os índices de marca sejam valorados mais favoravelmente quando acontece o processamento pré-atentivo, em detrimento de quando o processamento é atentivo.

H5: Os índices de marca são valorados mais favoravelmente quando ocorre o processamento préatentivo da revista do que quando o processamento é atentivo.

\section{MÉTODO DA PESQUISA}

Congruente com os trabalhos realizados na área, o estudo seguirá por uma abordagem hipotético-dedutiva, a fim de comparar o processamento pré-atentivo do atentivo em termos de registro de três marcas anunciantes de uma revista, testando, para isso, as memórias implícita e

REMark - Revista Brasileira de Marketing, São Paulo, v. 12, n. 4, p. 01-26, out./dez. 2013. 
explícita dos indivíduos e sua valoração de marcas. Dessa forma, basear-se-á em uma observação sistemática em condições experimentais cuidadosamente controladas em um laboratório, tendo como foco a validade interna de seus resultados (Herriot, 1977). Além disso, seguindo as orientações de Herriot (p.42, 1977), o propósito do experimento não será relevado aos seus participantes, já que "a melhor política é manter o sujeito na ignorância das finalidades do experimento". Os reais propósitos do estudo serão apresentados aos participantes somente ao término de seu procedimento (debriefing).

O experimento conta com um desenho fatorial 2 (Meio de divulgação: slide ou impresso) x 2 (Atenção: dirigida a revista como um todo, implicando processamento atentivo, ou focada somente nos textos, indicando processamento pré-atentivo). Foi informado aos 76 participantes, graduandos do curso de comunicação da Universidade de São Paulo (USP), que se tratava de uma pesquisa de mercado para testar a aceitação do público frente a uma nova revista que será lançada no mercado. Como a manipulação da atenção foi realizada de forma escrita, houve distribuição randômica dos participantes entre esses grupos. Abaixo, é apresentado o quadro com a distribuição dos participantes entre os grupos.

Tabela 1 - Distribuição dos Participantes entre os Tratamentos

\begin{tabular}{|l|c|c|}
\hline \multicolumn{1}{|c|}{ TRATAMENTO } & FREQUÊNCIA & PORCENTAGEM \\
\hline 1-Pré-atenção com revista mostrada em slide & 17 & $22 \%$ \\
\hline 2- Atenção com revista mostrada em slide & 19 & $25 \%$ \\
\hline 3 Pré-atenção com revista impressa & 19 & $25 \%$ \\
\hline 4- Atenção com revista impressa & 21 & $28 \%$ \\
\hline \multicolumn{1}{|c|}{ Total } & 76 & $100 \%$ \\
\hline
\end{tabular}

Fonte: Elaboração própria.

O experimento foi realizado em duas fases, seguindo as orientações de Lee (1994): de estudo, no qualé pedido para que os indivíduos folhassem as revistas de acordo com as instruções recebidas, enquanto são expostos a estímulos de forma pré-atentiva (e atentiva, no caso); e de teste, quando é pedido para que resgatem ou formulem julgamentos acerca desses estímulos.

Para a análise dos dados, foi utilizada a técnica estatística paramétrica de Análise de Variância de Um Fator (one-way ANOVA), com o teste Tukey como procedimento de comparação a posteriori (post hoc), atentando para o atendimento dos seus pressupostos metodológicos.

REMark - Revista Brasileira de Marketing, São Paulo, v. 12, n. 4, p. 01-26, out./dez. 2013. 


\subsection{MATERIAIS}

Três marcas foram desenvolvidas exclusivamente para esse estudo, desenvolvendo-se logomarcas completas, compostas de nome com grafismo, logotipo (sinal visual), slogan e apelo de venda. Optou-se por utilizar-se marcas fictícias, assim como não explicitar a categoria de produto a qual cada marca pertence, com o intuito de evitar possíveis interferências provenientes dessas informações, conforme relatado por estudos anteriores (Yoo, 2005). Para tanto, foi constituído um corpo de juízes, composto por sete profissionais, todos também com experiência e atuação na área de construção de marcas e identidade visual. Dessa forma, sem que houvesse a interação entre os juízes, foram criadas doze opções de marca, posteriormente colocadas em votação, o que resultou na seguinte seleção das marcas a serem utilizadas no estudo: Impacto (6 votos), Happen (4 votos) e Supremo (4 votos).

A mídia (impressa ou em slide) contou com oito páginas $(10,5 \times 15 \mathrm{~cm})$, preenchidas por capa, introdução e sumário, três matérias, encerramento e editorial. As logomarcas se situaram na introdução e em duas matérias.

\section{ANÁLISE DOS DADOS}

O questionário aplicado aos participantes era composto, basicamente, de cinco conjuntos principais: perfil da amostra; teste de atenção prestada; teste de memória explícita, com evocação livre e forçada e reconhecimento; teste de memória implícita, com efe ito priming (tarefa de completar palavras); e valoração das marcas.

A amostra foi composta de 76 graduandos do curso de Comunicação da Universidade de São Paulo (USP), metade deles cursando o primeiro ano (49\%), seguido por grande presença no terceiro ano (38\%). A amostra ainda se caracteriza por uma grande maioria de solteiros (97\%), com preponderância do sexo masculino (54\%) e idade média de 21 anos (min=17; máx=27). Metade dos participantes exercem atividade profissional além dos estudos (53\%), assim como também quase metade da amostra declarou possuir renda própria (43\%), no valor médio de R \$589/mês (mín=0; máx=4000). A maioria dos estudantes mora com os pais (59\%), que majoritariamente possui alta escolaridade, com ensino superior completo (76\% tanto pai quanto mãe), e mínimo de 4 salários mínimos, com a seguinte distribuição de clas ses sociais: C (36\%), B (33\%), A (26\%) e D ou E (5\%), segundo cálculo do IBGE.

REMark - Revista Brasileira de Marketing, São Paulo, v. 12, n. 4, p. 01-26, out./dez. 2013. 


\subsection{ATENÇÃO PRESTADA (TESTE DE MANIPULAÇÃO)}

A atenção prestada aos textos das matérias principais da revista foi medida de duas formas: livre atribuição dos participantes de quanto de atenção prestou, bem como em termos de acertos e erros às perguntas relacionadas a cada matéria (uma pergunta por matéria).

De modo geral, a maioria dos participantes afirmou ter prestado média atenção aos textos das matérias principais da revista $(55 \%)$, seguido por $22 \%$ que disseram ter prestado bastante atenção. Observou-se uma diferença significativa $(F(3,72)=3,12, p=0,031)$ entre os tratamentos 1Pré-atenção com revista mostrada em slide $(\mathrm{M}=3,18, \mathrm{SD}=0,8090)$ e 4-Atenção com revista impressa $(M=2,48, S D=1,03)$, ou seja, houve atribuição de maior nível de atenção prestada quando a revista mostrada em slide foi processada de forma pré-atentiva, em detrimento da revista impressa processada atentivamente.

A fim de se obter um panorama geral da atenção prestada às matérias da revista em termos de acertos e erros, as três questões referentes a cada matéria foram analisadas em conjunto $(\alpha=0,524)$. Dessa forma, encontrou-se uma média geral de acertos em $85 \%$ das respostas. Foram encontradas diferenças significativas $(\mathrm{F}(3,224)=5,62, \mathrm{p}=0,001)$ entre o grupo 2-Atenção com revista mostrada em slide $(\mathrm{M}=1,3, \mathrm{SD}=0,46)$ com os tratamentos 1-Pré-atenção com revista mostrada em slide $(M=1,06, S D=0,24)$ e 3-Pré-atenção com revista impressa $(M=1,07, S D=0,26)$. Também houve diferença significativa entre os tratamentos Pré-atenção com revista mostrada em slide $(\mathrm{M}=1,06, \mathrm{SD}=0,24)$ e 4-Atenção com revista impressa $(\mathrm{M}=1,17, \mathrm{SD}=0,38)$. Em todos os casos, observa-se uma preponderância de acertos nas questões referentes às matérias quando as revistas eram processadas pré-atentivamente, comparando quando o processamento atentivo das revistas ocorreu.

Tabela 2 - Resumo Análise Variável Atenção

\begin{tabular}{|c|c|c|}
\hline QUES TÃO & ANOVA & ANÁLISE DIFERENÇA \\
\hline $\begin{array}{c}\text { Livre Atribuição de } \\
\text { Atenção Prestada }\end{array}$ & $\mathrm{F}(3,72)=3,12$ & 1-Pré-atenção revista em slide > 4-Atenção revista impress a \\
\hline $\begin{array}{c}\text { Teste de Atenção } \\
\text { Geral (Erros e Acertos) } \\
\text { Três Matérias }\end{array}$ & $\mathrm{F}(3,224)=5,62$ & $\begin{array}{c}\text { 1-Pré-atenção revista em slide e 3-Pré-atenção revista impres sa }>\text { 2- } \\
\text { Atenção revis ta em slide; } \\
\text { 1-Pré-atenção revista em slide > 4-Atenção revista impress a }\end{array}$ \\
\hline
\end{tabular}

Fonte: Elaboração própria.

As questões referentes à atenção prestada servem como teste de manipulação da atenção dirigida às marcas anunciadas na revista. Quanto mais atenção for focada nos textos da revista, objeto-alvo de processamento, menor a capacidade do individuo de processar também de forma 
atentivamente as marcas dispostas em regiões perimetrais ao foco da atenção (Janisweski, 1993; Shapiro, Macinnis, \& Heckler, 1994). Dessa forma, conforme esperado, maiores níveis de atenção foram atribuídos ao processamento pré-atentivo quando comparado ao atentivo. Como consequência da maior atenção dirigida aos textos, também eram esperados mais acertos nas perguntas referentes às matérias no caso do processamento pré-atentivo, em detrimento do processamento atentivo. Com esses resultados, pode-se inferir que a manipulação das atenções prestadas pelos indivíduos funcionou conforme o planejado.

\subsection{MEMÓRIA EXPLÍCITA (H1)}

Entrando nas questões referentes à memória explícita dos indivíduos, foram testadas as capacidades de evocação (tanto de forma livre quanto forçada) e de reconhecimento das marcas anunciantes da revista.

A capacidade de livre evocação das marcas foi testada perguntando aos participantes quais marcas primeira e livremente lhe vinham à cabeça. Sendo assim, de forma geral, nenhuma marca foi evocada livremente em $96 \%$ dos casos. Ressalta-se aqui a baixíssima capacidade dos participantes de evocar livremente as marcas anunciantes em todos os tratamentos, sem diferenças significativas $(\mathrm{p}=0,489)$. É interessante pontuar também que quando a revista foi mostrada em slide, independente da atenção dirigida, nenhuma marca foi evocada, ao contrário de quando o meio de divulgação era impresso, que embora mínimo, respondeu por um percentual de livre evocação.

A evocação forçada das marcas anunciantes foi auferida pedindo para que os participantes listassem diretamente quais marcas apareciam expostas na revista. Dessa forma, a maioria dos participantes (60\%), de modo geral, não conseguiu evocar forçadamente nenhuma marca, seguido por $24 \%$ de casos no qual uma marca foi evocada, $12 \%$ das vezes em que duas marcas foram evocadas e $4 \%$ quando todas as três marcas foram evocadas. Comparando a quantidade de marcas evocadas com a quantidade total possível de evocação, encontrou-se um percentual de 19,7\% marcas evocadas. Foram obtidas diferenças significativas $(F(3,72)=6,08, p=0,001)$ entre os tratamentos 1-Pré-atenção com revista mostrada em slide $(\mathrm{M}=0,12, \mathrm{SD}=0,33)$ e 4-Atenção com revista impressa $(\mathrm{M}=0,19, \mathrm{SD}=0,89)$. Isto é, ainda que com baixos percentuais de evocação forçada das marcas anunciantes da revista, um maior índice de memória explícita (nesse quesito) é atribuído ao processamento atentivo, contra o processamento pré-atentivo, assim como ao meio impresso, quando comparado à revista mostrada em slide.

REMark - Revista Brasileira de Marketing, São Paulo, v. 12, n. 4, p. 01-26, out./dez. 2013. 


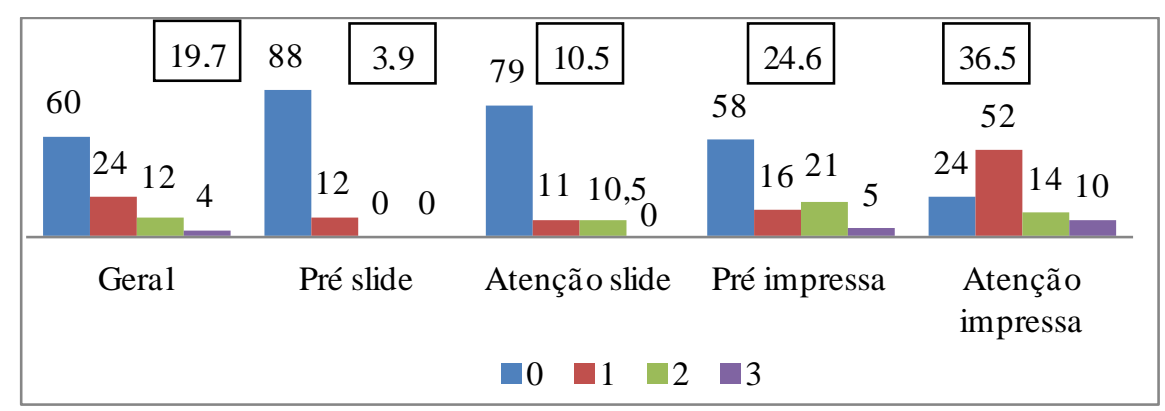

Figura 1: Marcas Evocadas Forçadamente (em \%)

Fonte: Elaboração própria.

A capacidade de reconhecimento das marcas anunciantes foi avaliada pedindo aos participantes para que selecionasse, frente três marcas distratoras, qual marca eles acreditavam ter visto anunciada na revis ta e qual o grau de certeza dessa resposta.

Analisando as três marcas de forma conjunta $(\alpha=0,832)$, teve-se um acerto geral no reconhecimento das marcas em $73,1 \%$ dos casos. Diferenças significativas $(F(3,193)=7,24$, $\mathrm{p}<0,0005)$ foram encontradas entre o tratamento 1-Pré-atenção com revista mostrada em slide $(\mathrm{M}=1,43, \mathrm{SD}=0,50)$ com 4-Atenção com revista impressa $(\mathrm{M}=1,13, \mathrm{SD}=0,34)$ e também 3-Préatenção com revista impressa $(\mathrm{M}=1,15, \mathrm{SD}=0,37)$; assim como entre o grupo 2-Atenção com revista mostrada em slide $(\mathrm{M}=1,41, \mathrm{SD}=0,49)$ e os tratamentos 3-Pré-atenção com revista impressa $(M=1,15 \mathrm{SD}=0,36)$ e também 4-Atenção com revista impressa $(\mathrm{M}=1,13, \mathrm{SD}=0,34)$. Os resultados mostram que o meio de divulgação da revista influencia na capacidade de reconhecimento das marcas pelos indivíduos, com as marcas sendo mais reconhecidas quando a revista era lida em meio impresso, tanto pré-atentiva quanto atentivamente, em detrimento de quando era mostrada em slide.

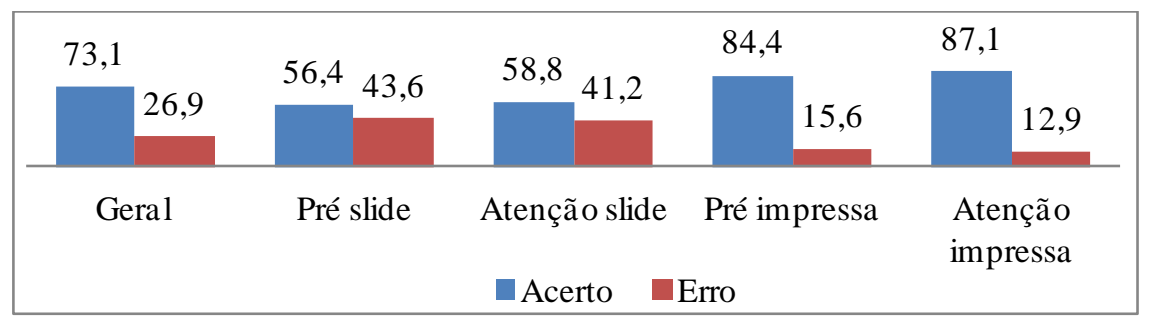

Figura 2: Capacidade de Reconhecimento (Acerto e Erro) Três Marcas (em \%)

Fonte: Elaboração própria.

Em relação ao grau de certeza no reconhecimento das três marcas, analisado conjuntamente, de forma geral, $38 \%$ dos participantes declararam estarem nada certos de suas respostas. Em termos de escala, a maioria das respostas se concentrou em menores graus de 
certeza $(53 \%)$, contra $31,5 \%$ das respostas em maiores graus de certeza $(15,5 \%$ se fixou na média). Foram encontradas diferenças significativas $(F(3,202)=12,9, \quad p<0,0005)$ entre os tratamentos: 2-Atenção com revista mostrada em slide $(\mathrm{M}=1,88, \mathrm{SD}=1,21)$ com 4-Atenção com revista impressa $(\mathrm{M}=3,32, \mathrm{SD}=1,41)$ e 3-Pré-atenção com revista impressa $(\mathrm{M}=2,85, \mathrm{SD}=1,61)$; 1-Pré-atenção com revista mostrada em slide $(\mathrm{M}=2,09, \mathrm{SD}=1,36)$ e 4-Atenção com revista impressa $(\mathrm{M}=3,32, \mathrm{SD}=1,41)$. Ressalta-se, aqui, uma preponderância do meio impresso comparado à revista mostrada em slide, com maiores graus de certeza atribuídos quando a revista era lida de forma impressa.

Analisando o cruzamento das respostas em que houve um correto reconhecimento das marcas com o grau de certeza desses acertos, de forma geral e conjunta, 30\% dos participantes que reconheceram acertadamente as marcas anunciantes declararam estarem nada certos de suas respostas, seguidos de $23 \%$ que afirmaram estarem absolutamente certos. Em termos de escala, $45 \%$ das respostas se concentraram em menores graus de certeza, contra $40 \%$ atribuídas a maiores graus de certeza ( $15 \%$ se concentrou na média). Evidenciaram-se diferenças significativas $(\mathrm{F}(3,140)=6,85, \mathrm{p}<0,0005)$ entre o tratamento 4-Atenção com revista impressa $(\mathrm{M}=3,46$, $\mathrm{SD}=1,38)$ e os grupos 1-Pré-atenção com revista mostrada em slide $(\mathrm{M}=2,04, \mathrm{SD}=1,46)$ e 2 Atenção com revista mostrada em slide $(\mathrm{M}=2,3, \mathrm{SD}=1,37)$. Semelhante ao grau de certeza geral, observou-se uma influência do meio de divulgação da revista no grau de certeza sentido pelos participantes quando eles reconheceram corretamente as marcas, com maiores graus de certeza declarados quando a revista lida era impressa, em vez de quando era mostrada em slide.

Tabela 3 - Resumo Análise Variável Memória Explícita

\begin{tabular}{|c|c|c|}
\hline QUES TÃO & ANOVA & ANÁLISE DIF ERENÇA \\
\hline Evocação Forçada & $F(3,72)=6,08$ & 4-Atenção revista impressa > 1-Pré-atenção revista em slide \\
\hline Reconhecimento Três Marcas & $\mathrm{F}(3,193)=7,24$ & $\begin{array}{l}\text { 3-Pré-atenção revista impressa e 4-Atenção revista impressa > } \\
\text { 1-Pré-atenção revista em slide; } \\
\text { 3-Pré-atenção revista impressa e 4-Atenção revista impressa > } \\
\text { 2-Atenção revista em slide }\end{array}$ \\
\hline Grau de Certeza & $\mathrm{F}(3,202)=12,9$ & $\begin{array}{l}\text { 4-Atenção revista impressa e 3-Pré-atenção revista impressa } \\
\text { > 2-Atenção revista em slide } \\
\text { 1-Pré-atenção revis ta em slide < 4-Atenção revista impressa. }\end{array}$ \\
\hline $\begin{array}{l}\text { Acerto Reconhecimento com } \\
\text { Grau de Certeza }\end{array}$ & $\mathrm{F}(3,140)=6,85$ & $\begin{array}{l}\text { 1-Pré-atenção revista em slide e 2- Atenção revista em slide > } \\
\text { 4-Atenção revista impressa }\end{array}$ \\
\hline
\end{tabular}

Fonte: Elaboração própria.

REMark - Revista Brasileira de Marketing, São Paulo, v. 12, n. 4, p. 01-26, out./dez. 2013. 
Com base nos resultados encontrados, pode-se afirmar que a memória explícita dos participantes acerca das marcas anunciantes da revista é influenciada pelo meio de divulgação dela, que interfere tanto na capacidade de evocação (livre e forçada) e de reconhecimento dos indivíduos, como no grau de certeza experienciado por eles. Além disso, foi observada também uma influência do modo de processamento na memória explícita dos participantes, com maiores índices de evocação forçada das marcas no caso do processamento atentivo, em vez do préatentivo. Esse resultado dá suporte à primeira hipótese do estudo, mostrando que, conforme a literatura sugere, a memória explícita é mais recorrente em casos de processamentos mais detalhados, que dirigem o foco da atenção e empregam capacidade cognitiva superior (Milosavljevic, 2007).

\subsection{MEMÓRIA IMPLÍCITA (H2)}

A memória implícita dos indivíduos foi testada por meio da averiguação do efeito priming, com a tarefa de completar palavras, similar ao jogo da velha. O procedimento adotado para a questão referente à tarefa de completar palavras (semelhante ao jogo da forca) foi desenvolvido seguindo as diretrizes utilizadas por outros pesquisadores da área (Duke \& Carlson, 1994; Yoo, 2005). Nesse sentido, foram pré-teste $(n=44) 39$ palavras sugeridas (28 em português e 11 em inglês) por um corpo de juízes como possíveis marcas distratoras, que propositalmente se assemelhassem às marcas-alvo. Foram escolhidas as palavras com taxa de sucesso na tarefa de completar palavras entre 15 e $46 \%$ das vezes, diretriz estabelecida e seguida por artigos da área (Duke \& Carlson, 1994; Yoo, 2005). Também as marcas-alvo desse estudo foram pré-testadas e se mantiveram dentro desse parâmetro. Como resultado desse procedimento, foram selecionadas onze palavras para serem completadas nessa tarefa ( 8 em português e 3 em inglês), contendo entre 6 a 8 letras, faltando 2 ou 3 dessas letras para que as palavras fossem completadas. Dessa forma, mais interessante que analisar estatisticamente a porcentagem de vezes em que as palavras foram completadas, comparando esses dados entre os grupos (sem diferença significativa, $\mathrm{p}=0,118$ ), deve-se confrontar as porcentagens resultantes com as esperadas inicialmente (prétestadas, entre $15 \%$ e $46 \%$ ).

Nesse sentido, comparando o total de marcas completadas com o total de chance possível de completar (\% entre realizado e esperado), foi obtido um nível de acerto geral de $85 \%$ de marcas completadas, muito superior à taxa encontrada no pré-teste para essas mesmas palavras. Além disso, de modo geral, uma marca foi completada $5 \%$ das vezes, duas marcas em $49 \%$ e as três marcas em $46 \%$ dos casos. Observa-se, também, que a melhor taxa de priming (maior \% de palavras completadas dentre as possíveis) foi encontrada no tratamento que combinava o processamento pré-atentivo com o meio de divulgação impresso.

REMark - Revista Brasileira de Marketing, São Paulo, v. 12, n. 4, p. 01-26, out./dez. 2013. 


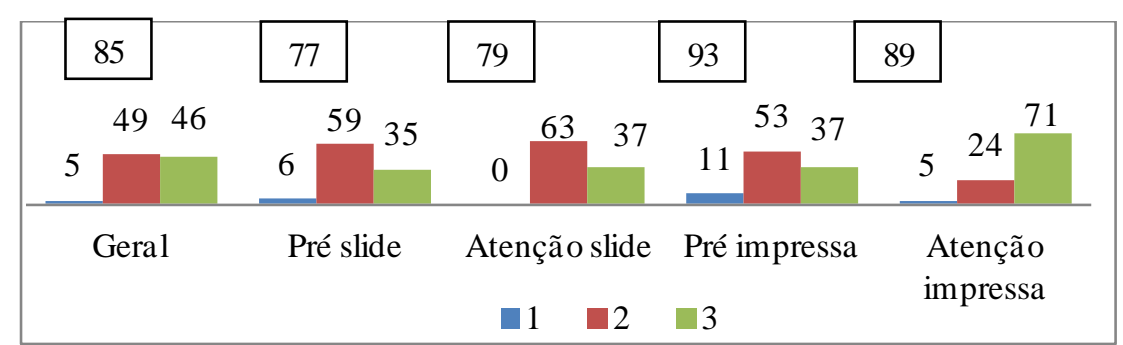

Figura 3: Marcas Completadas - Priming (em \%)

Fonte: Elaboração própria.

Combase nesses resultados, pode-se inferir que houve um efeito de priming experienciado pelos participantes: devido à prévia exposição às marcas, os participantes encontraram maior facilidade em completar as palavras presentes na tarefa com as marcas vistas anteriormente, incrementando de forma significativa o desempenho dos indivíduos na tarefa (Monin, 2002). Além disso, conforme a literatura sugere, esse tipo de memória foi encontrado nos dois tipos de processamento (Yoo, 2008), confirmando a segunda hipótese do trabalho.

\subsection{VALORAÇÃO DE MARCAS (H3, H4 e H5)}

A valoração da marca foi investigada em termos de positividade da marca e intenção de compra, mensuradas por meio de uma escala de diferencial semântico de cinco pontos (de 1, nada positiva/considerada em compras futuras, a 5, totalmente positiva/considerada em compras futuras).

Analisando a positividade das três marcas conjuntamente $(\alpha=0,642)$, tem-se o mesmo padrão de resultado, com grande concentração das respostas no ponto intermediário (49\%), além de uma tendência para maiores graus de positividade, correspondente a $29 \%$ das respostas, comparado a menores graus de positividade, que refletem $22 \%$ das respostas. Foram encontradas diferenças significativas $(\mathrm{F}(3,221)=3,58), \mathrm{p}=0,015)$ entre o tratamento 2-Atenção com revista em slide $(M=2,74, S D=1,03)$ com 3-Pré-atenção com revista impressa $(M=3,25, S D=0,95)$ e 4Atenção com revista impressa $(M=3,22, S D=0,76)$. Ou seja, além de uma preponderância do processamento pré-atentivo sobre o atentivo na valoração de positividade da marca, também foi observado uma influência do meio de divulgação da revista, com superioridade do meio impresso. Além disso, nota-se que a combinação do processamento pré-atentivo com o meio de divulgação impresso respondeu pela maior média de positividade das marcas.

REMark - Revista Brasileira de Marketing, São Paulo, v. 12, n. 4, p. 01-26, out./dez. 2013. 


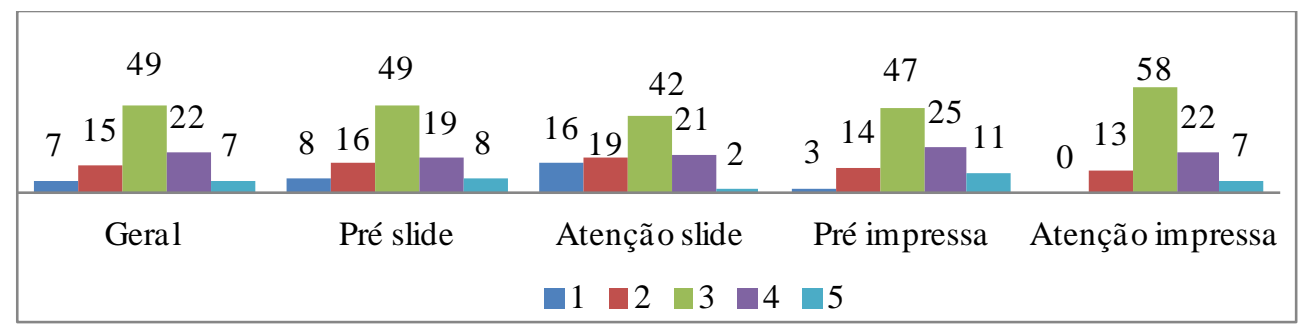

Figura 4: Escala (Diferencial Semântico) de Positividade Três Marcas (em \%)

Fonte: Elaboração própria.

Por terem sido expostos às marcas anteriormente, os participantes registram uma representação mental desses estímulos, que contribuem para os efeitos de fluência ou priming (Shapiro, Heckler, \& Macinnis, 1997), anteriormente discutidos. De acordo com a literatura, como resultado dos efeitos de fluência ou priming, o estímulo passa a ser valorado como mais positivo, mesmo que sem a intenção ou consciência por parte do indivíduo (Zajonc, 1968). No entanto, com a consciência da prévia exposição (ou seja, no caso do processamento atentivo), essa valoração positiva pode passar por um processo de correção, no qual a fluência experimentada pelos indivíduos é revisada e interpretada (Bornstein \& D’Agostinho, 1994). A pré-atenção, por se tratar de um processo inconsciente, não passa por esse processo de correção, permitindo que o estímulo continue a ser valorado como positivo (Shapiro, Heckler, \& Macinnis, 1997). Dessa forma, em conformidade com o previsto pela literatura, confirma-se a terceira hipótese do trabalho.

Considerando a intenção de compra das três marcas em conjunto $(\alpha=0,828)$, tem-se o mesmo padrão de resultado das análises das marcas isoladas: grande parte das respos tas atribuídas a menores graus de intenção de compra (46\%), seguido de uma concentração no ponto intermediário (39\%), além de outros $15 \%$ dispostos em maiores graus de intenção de compra. Diferenças significativas $(\mathrm{F}(3,224)=10,7, \mathrm{p}<0,0005)$ foram obtidas entre os tratamentos: 1-Préatenção com revista em slide $(\mathrm{M}=2,02, \mathrm{SD}=1,16)$ com 4-Atenção com revista impressa $(\mathrm{M}=2,7$, $\mathrm{SD}=1,03)$ e também com 3-Pré-atenção com revista impressa $(\mathrm{M}=2,89, \mathrm{SD}=0,92)$; e 2-Atenção com revista em slide $(M=2,03, S D=1,03)$ com 3-Pré-atenção com revista impressa $(M=2,89$, $\mathrm{SD}=0,92)$ e também com 4-Atenção com revista impressa $(\mathrm{M}=2,7, \mathrm{SD}=1,03)$. Mais uma vez, observa-se uma preponderância da influência do meio de divulgação da revista, com superioridade do meio impresso. Também no índice de intenção de compra a combinação do processamento préatentivo com o meio de divulgação impresso respondeu pela maior média na valoração das marcas.

REMark - Revista Brasileira de Marketing, São Paulo, v. 12, n. 4, p. 01-26, out./dez. 2013. 


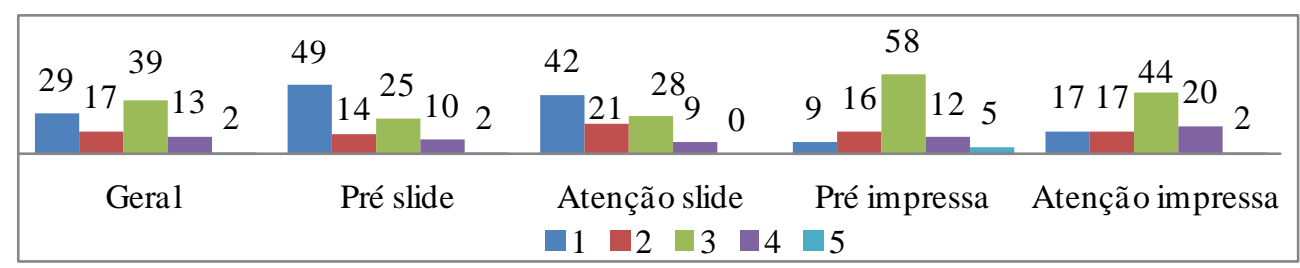

Figura 5: Escala (Diferencial Semântico) de Intenção de Compra Três Marcas (em \%)

Fonte: Elaboração própria.

Shapiro, Heckler e Macinnis (1997) afirmam que, com a prévia exposição, o estímulo é ativado na memória e seu acesso é facilitado, o que contribui para que esse estímulo seja incorporado no conjunto de consideração do indivíduo, ainda mais se esse processo for inconsciente. Ou seja, assim como no caso da valoração positiva, a prévia exposição (e os efeitos decorrentes dela) aumenta as chances de que o estímulo seja acatado em um futuro momento de decisão de compra, principalmente se essa inclusão não passe também por um processo de correção (McDonald \& Sharp, 2003). Nesse sentido, em consonância com os autores, foram encontrados maiores índices de intenção de compra das marcas quando houve o processamento pré-atentivo da revista do que quando o processamento foi atentivo, confirmando a quarta hipótese do trabalho.

Complementarmente, a valoração das marcas também foi analisada por meio de índices de marca, que agregaram conjuntamente atributos como já ter sido vista antes, familiaridade, positividade e intenção de compra, mensuradas por meio de uma escala Likert de cinco pontos (de 1 , pouco atributo a 5 , total atributo).

Em relação à primeira marca (Impacto), de forma conjunta $(\alpha=0,854)$ e geral, a maioria das respostas se concentrou em menores graus da escala (57\%), seguida de $22 \%$ das respostas dispostas no ponto intermediário e outras $21 \%$ atribuídas a maiores graus de escala. Foram encontradas diferenças significativas $(\mathrm{F}(3,291)=7,35), \mathrm{p}<0,0005)$ entre os tratamentos: 3-Préatenção com revista impressa $(\mathrm{M}=2,78, \mathrm{SD}=1,4)$ com 1-Pré-atenção com revista em slide $(\mathrm{M}=1,91, \mathrm{SD}=1,28)$ e 2-Atenção com revista em slide $(\mathrm{M}=2,05, \mathrm{SD}=1,28) ; 1$-Pré-atenção com revista em slide $(M=1,91, \mathrm{SD}=1,28)$ com 4-Atenção com revista impressa $(\mathrm{M}=2,57, \mathrm{SD}=1,2)$.

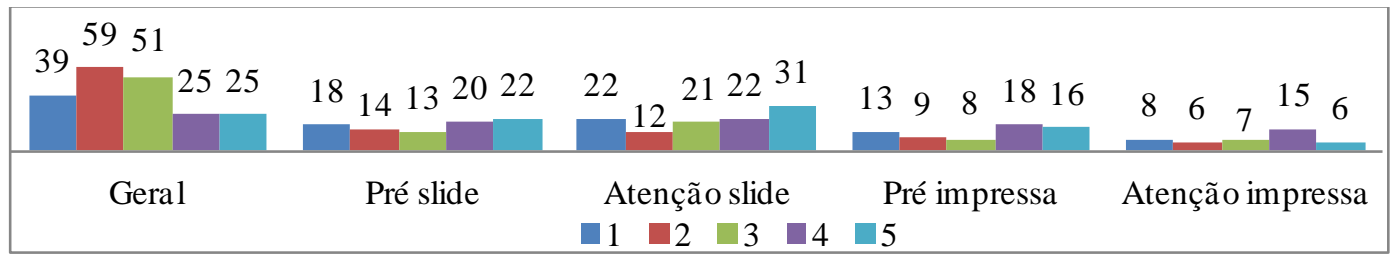

Figura 6: Escala (Likert) Índice Marca 1 - Impacto

Fonte: Elaboração própria.

REMark - Revista Brasileira de Marketing, São Paulo, v. 12, n. 4, p. 01-26, out./dez. 2013. 
O padrão de respostas se manteve para a segunda marca (Happen) que, de forma conjunta $(\alpha=0,791)$ e geral, concentrou a maioria das respostas em menores graus da escala (60\%), seguida de $23 \%$ das respostas dispostas no ponto intermediário e outras $17 \%$ atribuídas a maiores graus de escala. Diferenças significativas $(\mathrm{F}(3,291)=4,83), \mathrm{p}=0,003)$ foram obtidas entre o tratamento 1Pré-atenção com revista em slide $(\mathrm{M}=1,69, \mathrm{SD}=1,03)$ com os grupos 3-Pré-atenção com revista impressa (M=2,33, $\mathrm{SD}=1,2)$ e 4-A tenção com revista impressa $(\mathrm{M}=2,41, \mathrm{SD}=1,22)$.

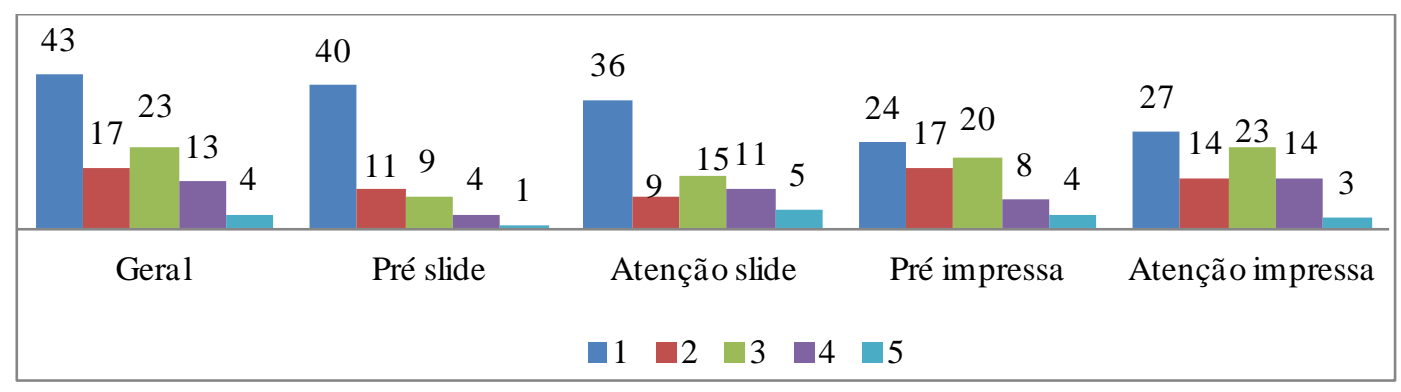

Figura 7: Escala (Likert) Índice Marca 2 - Happen

Fonte: Elaboração própria.

Da mesma forma, a terceira marca (Supremo), de forma conjunta $(\alpha=0,902)$ e geral, teve uma concentração de respostas em menores graus da escala (56\%), seguida de $28 \%$ das respostas dispostas no ponto intermediário e outras $16 \%$ atribuídas a maiores graus de escala. Por meio da análise ANOVA, foram encontradas diferenças significativas $(F(3,288)=4,78), p=0,003)$ entre o tratamento 3-Pré-atenção com revista impressa $(\mathrm{M}=2,59, \mathrm{SD}=1,29)$ com os grupos 1-Pré-atenção com revista em slide $(M=2, S D=1,31)$ e 2-Atenção com revista em slide $(M=1,96, S D=1,01)$.

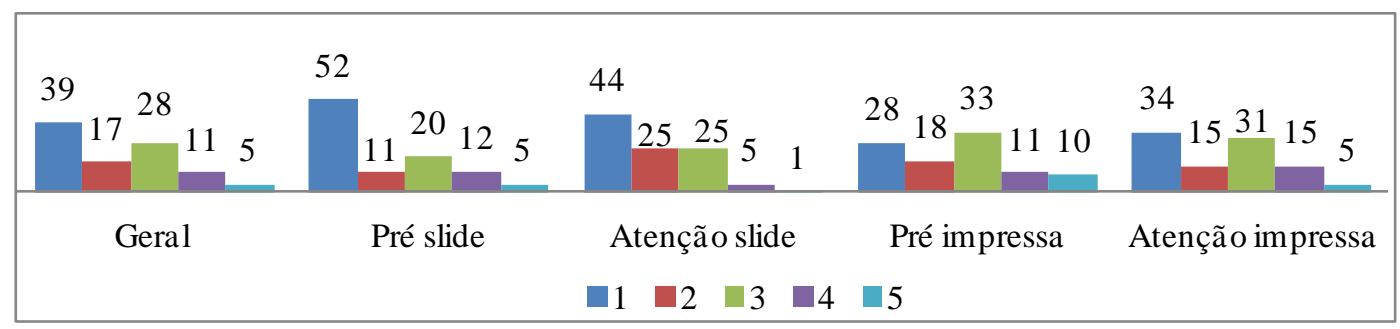

Figura 7: Escala (Likert) Índice Marca 2 - Happen

Fonte: Elaboração própria.

Devido à ausência de consciência acerca da prévia exposição, o indivíduo fica incapacitado de se engajar em um processo de correção da valoração positiva atribuída ao estímulo. Dessa forma, é defendido que, de maneira geral, o processamento dos estímulos de forma pré-atentiva consegue impactar mais os indivíduos receptores do que quando o processamento ocorre de modo atentivo (Bornstein \& D'Agostinho, 1994). Is so também foi encontrado no estudo, com os índices

REMark - Revista Brasileira de Marketing, São Paulo, v. 12, n. 4, p. 01-26, out./dez. 2013. 
das três marcas sendo valorados mais favoravelmente quando os indivíduos as processavam de forma pré-atentiva do que quando realizavam um processamento atentivo, confirmando a quinta hipótese traçada.

Tabela 4 - Resumo Análise Variável Valoração de Marca

\begin{tabular}{|c|c|l|}
\hline QUES TÃO & ANOVA & \multicolumn{1}{c|}{ ANÁLISE DIFERENÇA } \\
\hline Positividade & $\mathrm{F}(3,221)=3,58$ & $\begin{array}{l}\text { 3-Pré-atenção revista impressa e 4-Atenção revista impressa }>\text { 2- } \\
\text { Atenção revista em slide }\end{array}$ \\
\hline Intenção de Compra & $\mathrm{F}(3,224)=10,7$ & $\begin{array}{l}\text { 4-Atenção revista impressa e 3-Pré-atenção revista impressa }>\text { 1-Pré- } \\
\text { 4-Atenção revista impressa e 3-Pré-atenção revista impressa > 2- } \\
\text { Atenção revista em slide }\end{array}$ \\
\hline Índice Marca 1 & $\mathrm{F}(3,291)=7,35$ & $\begin{array}{l}\text { 3-Pré-atenção revista impressa > 1-Pré-atenção revista slide e 2- } \\
\text { 4-Atenção revista impressa > 1-Pré-atenção revista em slide }\end{array}$ \\
\hline Índice Marca 2 & $\mathrm{F}(3,291)=4,83$ & $\begin{array}{l}\text { 3-Pré-atenção revista impressa e 4-Atenção revista impressa > 1-Pré- } \\
\text { atenção revista em slide. }\end{array}$ \\
\hline Indice Marca 3 & $\mathrm{F}(3,288)=4,78$ & $\begin{array}{l}\text { 3-Pré-atenção revista impressa > 1-Pré-atenção revista slide e 2- } \\
\text { Atenção revista slide }\end{array}$ \\
\hline
\end{tabular}

Fonte: Elaboração própria.

Com base nos resultados encontrados, pode-se afirmar que a valoração das marcas anunciantes da revista tanto em termos de positividade e intenção de compra pelos indivíduos como em relação aos índices de marca sofre influência do meio de divulgação. Outra influência é o tipo de processamento, com maiores índices de valoração atribuídos ao processamento préatentivo, em detrimento do atentivo. Destaca-se também a preponderância na combinação entre o processamento pré-atentivo com o meio de divulgação impresso, respondendo por maiores índices em ambos os quesitos.

\section{CONSIDERAÇÕES FINAIS}

O registro das informações processadas pelo individuo depende da complexidade do processamento empregado, variando de acordo com a capacidade cognitiva destinada à tarefa: inferior, realizando apenas uma varredura rápida e geral do ambiente (pré-atenção) ou superior, direcionando e focando toda a atenção (Yoo, 2008). Dessa forma, esse registro das informações pode ocorrer de duas formas: memória explícita, cujo processo é consciente e intencional, fazendo 
referência direta ao passado, ou memória implícita, de natureza automática e inconsciente, sem retorno explícito do passado (Shapiro \& Krishnan, 2001).

Os resultados encontrados nesse estudo reforçam a teoria acerca das diferenças entre os registros das informações, com a confirmação das cinco hipóteses traçadas. Uma maior incidência de memória explícita foi observada no processamento atentivo. Segundo, em relação à memória implícita, os efeitos de fluência ou priming foram detectados em ambos os processamentos. Os efeitos na valoração de marca em termos de positividade e intenção de compra e também relação aos índices de marca (com os atributos de já ter visto antes, positiva, familiar e intenção de compra) foram mais fortes quando houve o processamento pré-atentivo do que quando o processamento foi atentivo, devido ao processo de correção consciente envolvido nesse último.

$\mathrm{O}$ estudo tem como principal contribuição evidenciar que diferentes níveis de processamento (de acordo com a atenção alocada) resultam em formas díspares de registro das informações processadas, influenciando também a forma como elas são percebidas e interpretadas. Mais especificamente, os resultados encontrados ressaltam a superioridade do processamento préatentivo na capacidade de influência dos indivíduos, respondendo por melhores índices no posterior processo de valoração dos estímulos, mesmo que os indivíduos falhem em evocar ou reconhecer esses estímulos (memória explícita).

Como principal implicação, destaca-se a importância de um adequado processo decisório em relação à emissão de propagandas, que deve considerar não só o meio de divulgação e as próprias características dos anúncios a fim de garantir que os objetivos dessa divulgação sejam atendidos. Ou seja, além do processo de emissão em si, deve-se considerar também o processo de recepção dessas emissões pelos indivíduos, levando em conta fatores como a disposição, a atenção e a capacidade de processamento dos receptores.

Além disso, o estudo encontrou também uma influência do meio de divulgação da mídia, com taxas superiores tanto de memória implícita como de explícita atribuídas ao meio impresso, em detrimento da divulgação em slide. Deve-se ressaltar que isso aconteceu mesmo quando os indivíduos estavam direcionando o foco de atenção à tarefa (processamento atentivo). Nesse sentido, pode-se estender esse resultado para outros campos, questionando-se a eficácia da utilização dos slides como meio de divulgação. No campo da educação, por exemplo, os slides são sabidamente bastante utilizados no meio acadêmico, principalmente em aulas universitárias, palestras e eventos acadêmicos. Com o resultado encontrado nesse estudo, pode-se questionar se o slide como instrumento de ensino é de fato o meio de divulgação mais adequado.

REMark - Revista Brasileira de Marketing, São Paulo, v. 12, n. 4, p. 01-26, out./dez. 2013. 


\section{REFERÊNCIAS}

Acar, A. (2007), Testing the Effects of Incidental Advertising Exposure in Online Gaming Environments. Journal of Interactive Advertising, 8(1), 45-56.

Baars, B.J. (1989), A Cognitive Theory of Consciousness. Cambrid ge University Press.

Bauman, Z. (2001), Modernidade Líquida. 1ª ed. Rio de Janeiro: J. Zahar Ed.

Bornstein, R., \& D'Agostino, P. R. (1992), Stimulus Recognition and the Mere Exposure Effect. Journal of Personality and Social Psychology, 63(4), 545-52.

Campbell, M. C., \& Keller, K. L. (2003), Brand Familiarity and Advertising Repetition Effects. Journal of Consumer Research, v.30.

De Weerd, P. (2003), Attention, neural basis of. In L. Nadel (Ed.), Encyclopedia of cognitive science (Vol. 1, pp. 238-246). London: Nature Publishing Group.

Duke, C. R., \& Carlson, L. B. (1994), Applying implicit memory measures: Word fragment completion in advertising tests. Journal of Current Issues and Research in Advertising, 16, 2940 .

Eysenck, M. W. (1995), Principles of Cognitive Psychology. Hove: Lawrence Earlbaum Associates. $\square \square$ Selective AttentionPsychology Review, 2(1) 27-30.

Fang, X., Singh, S., \& Ahluwalia, R. (2007), An Examination of Different Explanations for the Mere Exposure Effect. Journal of Consumer Research, 34.

Hauser, J. R., \& Wernerfelt, B. (1990), An Evaluation Cost Model of Consideration Sets. Journal of Consumer Research, v.16, n.4.

Herriot, P. (1977), Planejamento Experimental e Estatística. São Paulo: Zahar.

Holden, S. J., \& Vanhuele, M. Know the Name, Forget the Exposure: brand familiarity versus memory of exposure context. Psychology \& Marketing, v.16(6), p.479-496.

James, W. (1890), The principles of psychology. New York: Dover.

Janiszewski, C. (1993), Preattentive Mere Exposure Effects. Journal of Consumer Research, 20, 376-392.

Lee, A. Y. (1994), The Mere Exposure effect: is it a mere case of misattribution? Advances in Consumer Research, v.21, Association for Consumer Research, 270-275.

REMark - Revista Brasileira de Marketing, São Paulo, v. 12, n. 4, p. 01-26, out./dez. 2013. 
(2001), The Mere Exposure Effect $\square$ : An Uncertainty Reduction Explanation Revisited. Society for Personality and Social Psychology, 27, 1255-1266.

, \& Labroo, A. A. (2004), The Effect of Conceptual and Perceptual Fluency on Brand Evaluation. Journal of Marketing Research, 41(2), 151-165.

Logan, G. D. (1992), Attention and preattention in theories of automaticity. American Journal of Psychology, 105, 317-339.

Mcdonald, E., \& Sharp, B. (2003), Management perceptions of the importance of the Brand Awareness as an Indication of Advertising Effectiveness. Marketing Bulletin, 14, 1-15, Article 2.

Mandler, G., Nakamura, Y., \& Van Zandt, B. J. (1987), Nonspecific Effects of Exposure to Stimuli that cannot be Recognized. Journal of Experimental Psychology: Learning, Memory, \& Cognition, 13, 646-648.

Milosavljevic, M. (2007), The effects of preattention in an online advertising context: A neuro science perspective. Doutorado em Filosofia, Universidade de Florida.

\& CERF, M. (2008), First attention then intention: Insights from computational neuroscience of vision. International Journal of Advertising, 27(3), 381-398.

Monin, B. (2002), The Warm Glow Heuristic: When Liking Leads to Familiarity. Dissertation Abstracts International: Section B: The Sciences and Engineering, 62 (June).

Reilly, M., \& Parkinson, T. L. (1985), Individual and Product Correlates of Evoked Set Size for Consumer Package Goods. Advances in Consumer Research, 12, 492-497.

Rodrigues, A., Jablonski, B., \& Assmar, E. M. L. (2008), Psicologia Social. São Paulo: Editora Vozes.

Rossini, J. C., \& Galera, C. (2008), Seleção e Análise de Estímulos na Tarefa de Busca Visual. Psicologia: Reflexão e Crítica, 21(1), 20-27.

Shapiro, S., Macinnis, D. J., \& Heckler, S. E. (1994), Measuring and Assessing the Impact of Preattentive Processing on Ad and Brand Attitudes. In Measuring Advertising Effectiveness, Willians D. Wellls, p.27-44. Hilsdale: Ed. Lawrence Erlbaum.

(1997), The Effects of Incidental Ad Exposure on the Formation of Consideration Sets. Journal of Consumer Research, 24(1), 94-104.

(1999), When an Ad's Influence is Beyond Our Conscious Control: Perceptual and Conceptual Fluency Effects Caused By Incidental Ad $\square$ Exposure.Journal of Consumer Research, v.26.

REMark - Revista Brasileira de Marketing, São Paulo, v. 12, n. 4, p. 01-26, out./dez. 2013. 
, \& Krishnan, S. (2001), Memory based Measures for Assessing Advertising Effects: A Comparison of Explicit and Implicit Memory Effects. The Journal of Advertising, 30, p.114.

Sternberg, R. J. (2000), Psicologia Cognitiva. Porto Alegre: Artes Médicas.

Treisman, A., \& Gelade, G. A (1980). A feature Integration Theory of Attention. Cognitive Psychology, 12, 97-136.

Tversky, A., \& Kahneman, D. (1974), Judgment under Uncertainty: Heuristics and Biases. Science (New York, N.Y.), 185(4157), 1124-31.

Wolfe, J. M., \& Horowitz, T. S. (2003), Memory for Rejected Distracters in Visual Search? Visual Cognition, 10, p.257-298.

Yoo, C. (2005), Preattentive Processing of Web Advertising. Dissertação, Doutorado em Filosofia, Universidade de Texas, 1-207.

(2008), Unconscious Processing of Web Advertising: effects on implicit memory, attitude toward the brand, and consideration set. Journal of Interactive Marketing, 22(2), 218.

Zajonc, R. B. (1968), Attitudinal Effects of Mere Exposure. Journal of Personality and Social Psychology Monographs, 9(2, Pt. 2), 1-27.

Data do recebimento do artigo: 03/07/2013

Data do aceite de publicação: 24/10/2013

REMark - Revista Brasileira de Marketing, São Paulo, v. 12, n. 4, p. 01-26, out./dez. 2013. 